\title{
Infiltration of thymidine phosphorylase-positive macrophages is closely associated with tumor angiogenesis and survival in intestinal type gastric cancer
}

\author{
AKIHIKO KAWAHARA ${ }^{1,2}$, SATOSHI HATTORI ${ }^{3}$, JUN AKIBA ${ }^{4}$, KAZUTAKA NAKASHIMA $^{1,2}$, TOMOKI TAIRA ${ }^{1}$, \\ KOSUKE WATARI ${ }^{6}$, FUMIHITO HOSOI ${ }^{2,6}$, MANAMI UBA ${ }^{6}$, YUJI BASAKI ${ }^{6}$, KIKUO KOUFUJI ${ }^{5}$, \\ KAZUO SHIROUZU ${ }^{5}$, SHIN-ICHI AKIYAMA ${ }^{8}$, MICHIHIKO KUWANO ${ }^{2,7}$, \\ MASAYOSHI KAGE ${ }^{1,2}$ and MAYUMI ONO ${ }^{2,6}$

\begin{abstract}
${ }^{1}$ Department of Diagnostic Pathology, Kurume University Hospital; ${ }^{2}$ Center for Innovative Cancer Therapy and ${ }^{3}$ Biostatistics Center, Kurume University; Departments of ${ }^{4}$ Pathology and ${ }^{5}$ Surgery, Kurume University School of Medicine, Kurume; ${ }^{6}$ Department of Pharmaceutical Oncology, ${ }^{7}$ Laboratory of Molecular Cancer Biology, Department of Clinical Pharmaceutics, Graduate School of Pharmaceutical Sciences, Kyushu University, Fukuoka; ${ }^{8}$ Department of Medical Oncology, Institute of Health Biosciences, The University of Tokushima Graduate School, Tokushima, Japan
\end{abstract}

Received March 8, 2010; Accepted May 21, 2010

DOI: $10.3892 /$ or_00000873

\begin{abstract}
Thymidine phosphorylase (TP), an enzyme catalyzing the reversible phospholysis of thymidine, deoxyuridine and their analogs at their respective bases and 2-deoxyribose-1-phosphate, thus promoting angiogenesis, is often expressed in macrophages present in tumor stroma. In this study, we investigated whether infiltration of TP-positive macrophages as well as tumor-associated macrophages affected tumor angiogenesis. TP was expressed in human macrophage-like cells, but not in gastric cancer cells in culture. The expression level of TP, the number of infiltrating $\mathrm{CD}^{2} 8^{+}$and $\mathrm{CD} 163^{+}$macrophages, and microvessel density (MVD) in the tumor were further analyzed by immunohistochemistry in 111 patients with gastric cancer. Biostatistical analysis of digitized data obtained by image analysis showed that TP expression was significantly correlated with the number of infiltrating macrophages and MVD in intestinal type gastric cancer $(\mathrm{p}<0.05)$. The number of infiltrating macrophages was also correlated with MVD in both the intestinal and diffuse types $(\mathrm{p}<0.05)$. An increased number of $\mathrm{CD}^{+} 8^{+}$ macrophages was significantly associated with poor outcome in patients with intestinal type $(\mathrm{p}<0.001)$, but not diffuse type cancer. TP could be a specific marker enzyme that is expressed in tumor-infiltrating macrophages, being associated with tumor
\end{abstract}

Correspondence to: Dr Akihiko Kawahara, Department of Diagnostic Pathology, Kurume University Hospital, 67 Asahi-machi, Kurume 830-0011, Japan

E-mail: akihiko4@med.kurume-u.ac.jp

Key words: thymidine phosphorylase, tumor-associated macrophages, gastric cancer, tumor angiogenesis angiogenesis and poor prognosis in patients with intestinaltype gastric cancer.

\section{Introduction}

Thymidine phosphorylase (TP) is an enzyme catalyzing the reversible phospholysis of thymidine, deoxyuridine and their analogs at their respective bases, and 2-deoxyribose-1phosphate, thus playing a role in angiogenesis (1). The angiogenic activity of TP is due in part to production of reactive oxygen species by 2-deoxy-D-ribose, leading to up-regulation of angiogenic factors such as vascular endothelial growth factor and interleukin-8/CXCL8 through induction of oxidative stress and inflammatory signaling (2-4). TP is more highly expressed in differentiated than in undifferentiated gastric adenocarcinoma and patients whose tumors are TP-positive have a poorer prognosis than those with TP-negative tumors (5). Tumor expression of TP is significantly correlated with angiogenesis and invasion/metastasis in cancers of the colon and rectum, stomach, pancreas and bladder (6-9). We have previously observed a marked increase of TP expression in human macrophages in culture through transcriptional activation in response to inflammatory cytokines (10). We have also observed that in melanoma, the number of infiltrating macrophages increases depending upon invasion depth, in close association with angiogenesis (11), and that these macrophages are positive for TP expression (12). Shimaoka et al have further reported that TP expression in macrophages is correlated with the development of tumor microvessels, and also that TP plays an important role in tumor invasiveness and progression in differentiated gastric adenocarcinoma (8).

On the other hand, infiltration of macrophages in the tumor stroma often promotes the malignant progression of cancer through interaction with cancer cells $(13,14)$. Among such macrophages, those activated by bacterial products and 
interferon $\gamma$, known as inflammatory M1 type macrophages, have an IL-2 $2^{\text {high }}$, IN-23 $3^{\text {high }}$, IL-10 ${ }^{\text {low }}$ phenotype and produce NO, IL-1ß, TNF- $\alpha$ and IL-6. By contrast, anti-inflammatory and activated macrophages, known as M2 type macrophages, have an IL-12 $2^{\text {low }}$, IL-23 $3^{\text {low }}$, IL-10 $0^{\text {high }}$ phenotype and produce proangiogenic factors and various receptors such as scavenger receptors and mannose receptors $(15,16)$. These tumorsupporting macrophages are known as tumor-associated macrophages (TAMs), and support the processes of invasion, metastasis and angiogenesis by the production of inflammatory cytokines, chemokines, proteases, prostanoids, growth factors and angiogenic factors $(13,14)$, suggesting that TAMs are rather more closely linked to the M2 type than to the M1 type. Clinical studies have demonstrated a close association between the abundance of TAMs and poor prognosis or tumor angiogenesis in colon cancer and glioma $(17,18)$ as well as various other types of solid tumor including cancers of the breast, bladder and cervix (19). Various cytokines and growth factors are instrumental in the recruitment and accumulation of macrophages in the tumor stroma, and these macrophages then play a key role in the angiogenesis and malignant transformation (20). Depletion of these macrophages/TAMs by macrophage-targeting bisphosphonate encapsulated in liposomes markedly inhibits tumor growth, angiogenesis, and bone metastasis (21-23), thus corroborating the involvement of macrophages in these cancerrelated activities (14). Tumor-supporting macrophages such as TAMs can often be differentially characterized from tumor-suppressive macrophages. Tumor-supportive macrophages are active in extracellular matrix remodeling, tissue repair and angiogenesis, whereas tumor-suppressive macrophages are active in antimicrobial and antitumor activities through immunostimulatory functions (24-26). In patients with gliomas we have previously reported that the number of macrophages in stage IV glioblastoma is higher than that in grade II or III glioma, and is also closely correlated with microvessel density in the tumors (27).

Concerning the role of macrophages/TAMs in gastric cancer, infiltrating macrophages have been associated with a good prognosis, suggesting a beneficial effect of TAM aggregation in advanced gastric cancer (28). However, it has not been determined whether the effect of TAMs is dependent on the histological type of gastric cancer. Migita et al have reported that the number of macrophages along the invasive margin is significantly greater in patients without, than in those with, liver metastasis (29). Although no significant correlation has been observed between the number of macrophages and the depth of cancer invasion or lymph node metastasis, a significant association between macrophage number and the survival of patients with intestinal type gastric cancer has been reported (29). With regard to the classification of gastric cancer (30), it has been reported that the intestinal type tends to metastasize through blood vessels, whereas the diffuse type tends to cause peritoneal dissemination and/or lymph node metastasis (31). However, it still remains unknown whether tumor-supporting macrophages such as TAMs play a role in the malignant progression of gastric cancer in relation to histological type.

In the present study, we screened the expression of TP in human cancer cell lines and macrophage-like cell lines by
Western blot analysis. Our aim was to determine whether TP could be a specific biomarker of infiltrating macrophages in gastric cancer, and also whether such macrophages could affect angiogenesis, lymph node metastasis and peritoneal dissemination, as well as patient survival. We also discuss the possible relationship of TP-expressing macrophages with tumor angiogenesis and other malignant characteristics.

\section{Materials and methods}

Cell culture. The human gastric cancer cell lines MKN-28, MKN-45, MKN-74, AZ-521, NUGC-3 and KATO-III were cultured with RPMI supplemented with $10 \%$ fetal bovine serum (FBS) and incubated in humidified atmosphere of $5 \% \mathrm{CO}_{2}$ at $37^{\circ} \mathrm{C}$. The macrophage cell lines THP-1 and U937 were purchased from American Type Culture Collection (Manassas, VA, USA) and cultured in RPMI supplemented with $10 \%$ FBS and incubated in humidified atmosphere of $5 \% \mathrm{CO}_{2}$ at $37^{\circ} \mathrm{C}$. We also used $\mathrm{KB} / \mathrm{TP}$ expressing high amount of TP $(3,4)$.

Western blot analysis. Cells were rinsed with ice-cold PBS and lysed in buffer containing $50 \mathrm{mmol} / \mathrm{l}$ Tris- $\mathrm{HCl}, 350 \mathrm{mmol} / \mathrm{l}$ $\mathrm{NaCl}, 0.1 \% \mathrm{NP} 40,5 \mathrm{mmol} / \mathrm{l}$ EDTA, $50 \mathrm{mmol} / \mathrm{l} \mathrm{NaF}, 1 \mathrm{mmol} /$ 1 phenylmethylsulfonyl fluoride, $10 \mu \mathrm{g} / \mathrm{ml}$ aprotinin, $10 \mu \mathrm{g} / \mathrm{ml}$ leupeptin and $1 \mathrm{mmol} / \mathrm{Na}_{3} \mathrm{VO}_{4}$. Cell lysates were subjected to SDS-PAGE and blotted onto Immobilon membranes (Millipore Corp., Bedford, MA) as described previously. After transfer, the membrane was incubated with blocking solution followed by primary antibody. Antibody detection was done using an enhanced chemiluminescence system (Amersham Biosciences Corp., Piscataway, NJ). The intensity of the luminescence was quantified using a CCD camera combined with an image analysis system (LAS-1000; Fuji Film, Japan).

Patients and tumor samples. We examined 111 patients with advanced gastric cancer whose tumors had been surgically removed in the Department of Surgery of Kurume University, between 2001 and 2004. The age of the gastric cancer patients ranged from 33 to 86 years (median, 69), 77 were men and 34 were women. Histological types were performed according to the criteria of Lauren classification (21), and tumor stage was performed according to the TNM classification. Patient characteristics are summarized in Table I. Cancer stages were $18.0 \%$ Stage I (IA+IB), $14.4 \%$ Stage II, 22.5\% Stage III (IIIA+IIIB) and $45.0 \%$ Stage IV. At the time of surgery, 73 (65.8\%), $17(15.3 \%)$ and $26(23.4 \%)$ patients had lymph node metastasis, liver metastasis and peritoneal dissemination, respectively. No patients had been administered drugs before surgery, and the standard chemotherapy was performed after surgery: Stage II or III patients were administered by TS-1, and Stage IV patients were administered by a combination of TS-1 and cisplatin.

Immunohistochemistry (IHC) analysis. Paraffin-embedded tissue samples were cut at $4 \mu \mathrm{m}$ and examined on a coated slide glass and labeled with the following antibodies using the BenchMark XT (Ventana Automated Systems, Inc., Tucson, AZ, USA) and ChemMate Envision methods 
Table I. Clinicopathological characteristics in gastric cancer patients.

\begin{tabular}{|c|c|c|c|c|}
\hline & \multicolumn{4}{|c|}{ Histological type } \\
\hline & \multicolumn{2}{|c|}{$\begin{array}{c}\text { Total }(\%) \\
(\mathrm{n}=111)\end{array}$} & $\begin{array}{l}\text { Intestinal } \\
(\mathrm{n}=57)\end{array}$ & $\begin{array}{l}\text { Diffuse } \\
(n=54)\end{array}$ \\
\hline \multicolumn{5}{|l|}{ Age } \\
\hline Median (min-max) & 69 & $(33-86)$ & $67.5(33-86)$ & $69(39-85)$ \\
\hline \multicolumn{5}{|l|}{ Gender } \\
\hline Male & 77 & $(69.4)$ & 47 & 30 \\
\hline Female & 34 & $(30.6)$ & 10 & 24 \\
\hline \multicolumn{5}{|l|}{ Stage } \\
\hline I & 20 & $(18.0)$ & 11 & 9 \\
\hline II & 16 & $(14.4)$ & 9 & 7 \\
\hline III & 25 & $(22.5)$ & 13 & 12 \\
\hline IV & 50 & $(45.0)$ & 24 & 26 \\
\hline \multicolumn{5}{|l|}{$\begin{array}{l}\text { Lymph node } \\
\text { metastasis }\end{array}$} \\
\hline Present & 73 & $(65.8)$ & 37 & 36 \\
\hline Absent & 38 & $(34.2)$ & 20 & 18 \\
\hline \multicolumn{5}{|l|}{ Liver metastasis } \\
\hline Present & 17 & $(15.3)$ & 14 & 3 \\
\hline Absent & 94 & $(84.7)$ & 43 & 51 \\
\hline \multicolumn{5}{|l|}{$\begin{array}{l}\text { Peritoneal } \\
\text { dissemination }\end{array}$} \\
\hline Present & 26 & $(23.4)$ & 7 & 19 \\
\hline Absent & 85 & $(76.6)$ & 50 & 35 \\
\hline
\end{tabular}

(DakoCytomation, Glostrup, Denmark), TP (x2000, antibodies supplied by Taiho Pharmaceutical Co., Ltd., Tokyo Japan), CD68 (x1200, KP-1, DakoCytomation), CD163 (x100, Novocastra, Newcastle, UK) and CD34 (x200, Novocastra). The ChemMate Envision method was used for TP, CD68 and CD34. Endogenous peroxidase activity was inhibited by incubating the slides in $3 \% \mathrm{H}_{2} \mathrm{O}_{2}$ for $5 \mathrm{~min}$. CD68 and CD34 antigen retrieval was performed by treating with proteinase $\mathrm{K}$ for $5 \mathrm{~min}$. Each slide was incubated for $30 \mathrm{~min}$ with the antibody at room temperature. For staining detection, the ChemMate Envision method was used with DAB as chromogen. For CD163, BenchMark XT was used. This automated system used the streptavidin biotin complex method with DAB as chromogen (Ventana iView DAB Detection kit).

IHC expression area analysis for TP, infiltration macrophages and MVD assessment. We extracted the digital data of the expression using the following image analysis systems. TP, CD68, CD163 and CD34-stained specimens were examined to identify the areas with the high density. Images of the expression area were selected for clarity from 6 fields at x200 for each IHC specimen, using a CCD digital camera (Nikon, DXM1200). Expression analysis was performed to measure the expression area of TP, number of macrophages a

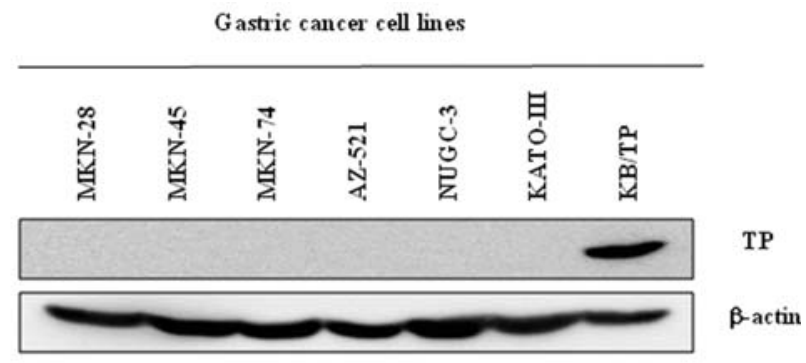

b

THP-1

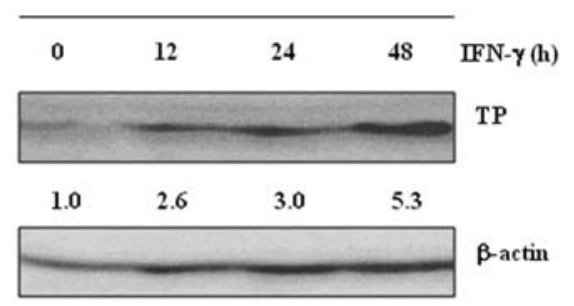

c

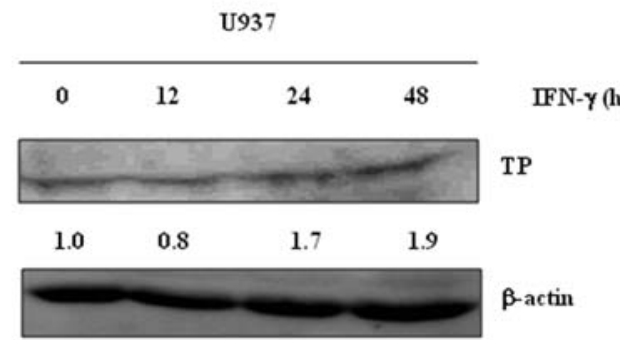

Figure 1. Expression levels of TP in 6 human gastric cancer cell lines (a) and 2 human macrophage-like cell lines $(b, c)$ in culture. Macrophages were treated with $50 \mathrm{U} / \mathrm{ml}$ of IFN $\gamma$. Western blot analysis of TP by specific antibody against TP was performed. Protein $(100 \mu \mathrm{g})$ of total cell lysates was loaded. Quantitative analysis for TP expression is presented when TP level in macrophages treated with INF $\gamma$ for $0 \mathrm{~h}$ is normalized as 1.0.

and MVD in all cases, using 'Win ROOF' (version 5.7; Mitani Corp., Osaka, Japan) computer software. The digitized data of the expression area were measured and averaged. The expression of TP often showed not only cancer cells, but also cancer stroma cells, such as macrophages, fibroblasts or vessels. The expression of TP was investigated, including both cancer cells and stroma cells.

Determination of co-expression of TP and CD68. We extracted the digital data of the expression levels using the following image analysis systems in all IHC specimens. Co-expression of TP and CD68 was revealed by the Nuance System (Cambridge Research and Instrumentation Inc., Woburn, MA, USA) (32). Double-stained slides for TP and CD68 antibodies were imaged at x200 through a liquid crystal tunable filter. TP and CD68 were labeled with a very intensely purple (VIP) substrate kit (Vector Laboratories, Burlingame, CA, USA) and 

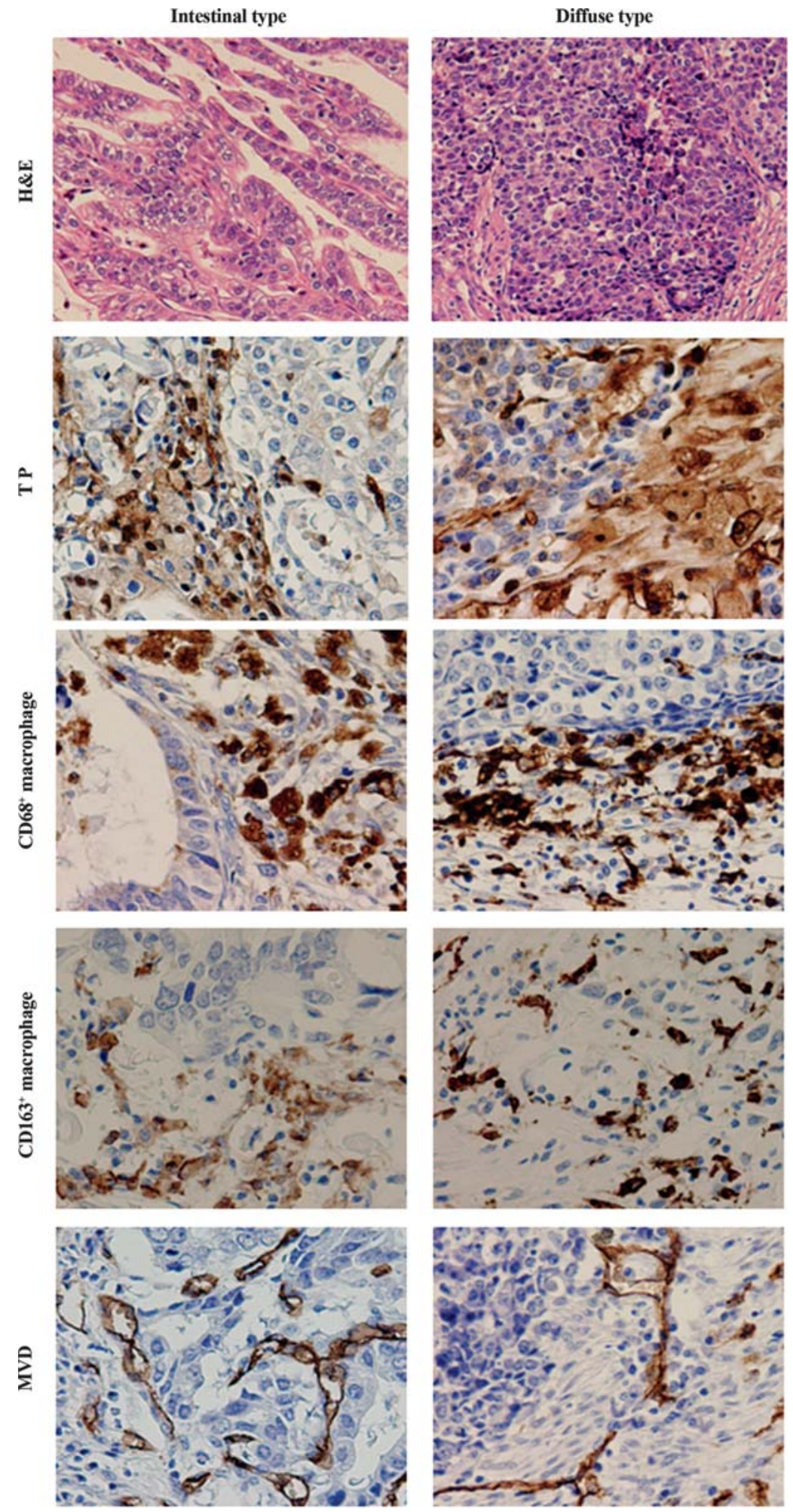

Figure 2. Representative images of immunohistochemical analysis of gastric cancer patients with intestinal and diffuse type (x200). Expression of TP, CD68, CD163 and CD34 is observed in cancer stroma in both histological types.

DAB as chromogen, respectively. Counterstaining was with hematoxylin. Multispectral image data were acquired from 420 to $720 \mathrm{~nm}$ at $\mathrm{x} 200$ in 3 fields from double-stained slides of 10 patients selected at random. Spectral unmixing was accomplished using Nuance software v2.8 and pure spectral libraries of individual chromogens. Expression areas of TP, CD68 and TP-CD68 co-expression were evaluated using Nuance software.
Statistical analysis. Associations among TP, CD68, CD163 and CD34 were evaluated using Spearman's rank correlation coefficients. The overall survival was defined as days from surgery until death due to any cause. Log-rank tests and Kaplan-Meier methods were applied to examine the effect of TP (CD68, CD163 and CD34) on overall survival classifying patients into negative and positive according to their TP (CD68, CD163 and CD34) were less than median or equal 


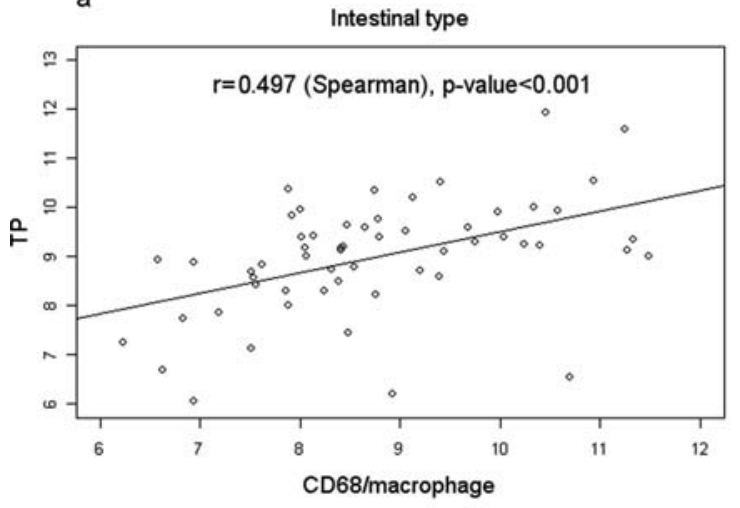

b

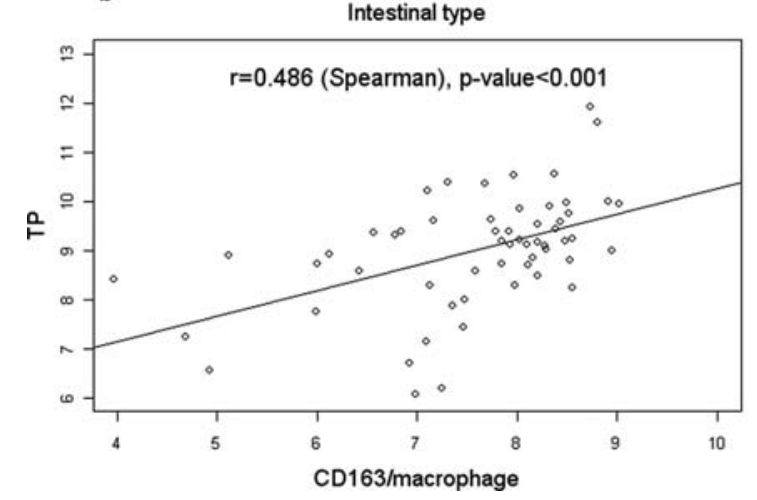

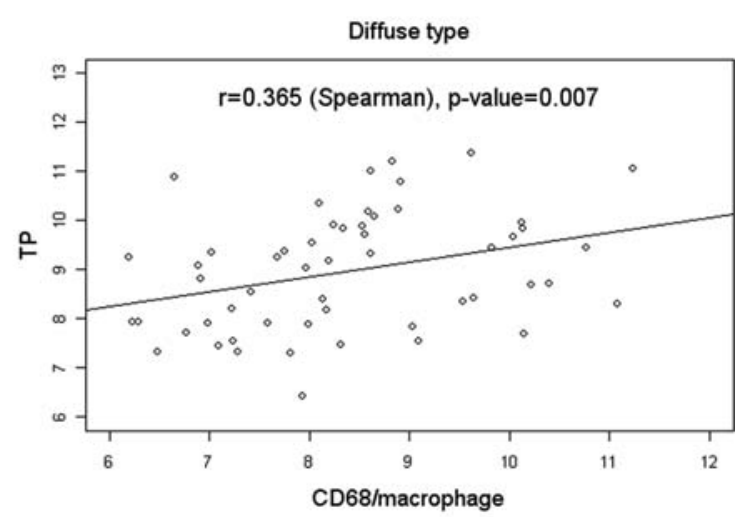

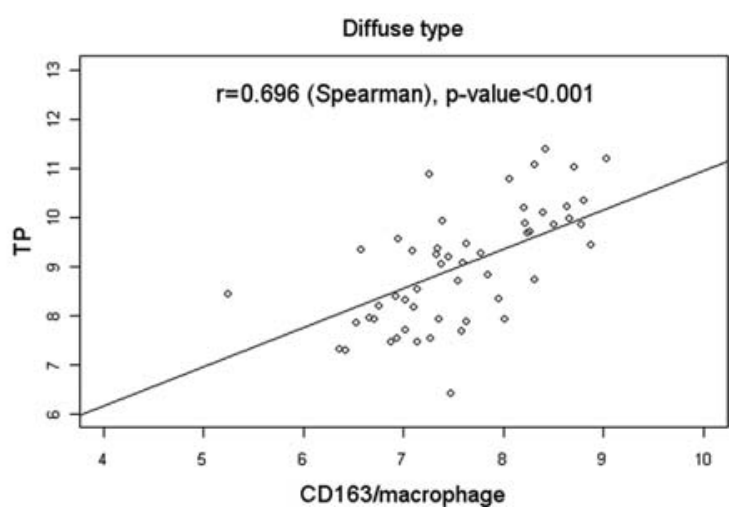

Figure 3. Correlation with TP and infiltrating macrophages (CD68 and CD163) in intestinal type (a) and diffused type (b) of gastric cancer.

to or more than median respectively. In addition, the Cox proportional hazards models were applied to examine the effect of TP (CD68, CD163 and CD34) on overall survival adjusting for possible confounding factors. The effects of TP (CD68, CD163 and CD34) on lymph node metastasis, liver metastasis and peritoneal dissemination were examined by logistic regression. In logistic regression analysis and Cox regression analysis, TP, CD68, CD163 and CD34 were not categorized into two categories of negative and positive and were log-transformed since they were highly skewed. When adjusting for possible confounding factors, Stage was not adjusted for since it might be an intermediate variable between TP (CD68, CD163 and CD34) and overall survival (33). Statistical analysis was performed by SAS version 9.1 (SAS Institute Inc., Cary, NC), StatXact (Cytel Inc., Cambridge, MA) and R version 2.8.1.

\section{Results}

Expression of TP in macrophage-like cell lines. TP is known to be expressed in both cancer cells and macrophages in the tumor stroma of gastric cancer (4). We first examined the expression of TP in human gastric cancer cell lines and macrophage-like cells in culture. Western blot analysis demonstrated no apparent expression of TP in any of the gastric cancer cell lines (Fig. 1a). By contrast, macrophage-like THP-1 and U937 cells expressed TP. Expression of TP was further stimulated by the inflammatory cytokine interferon $\gamma$ (Fig. 1b and c). TP was expressed rather specifically in macrophages relative to its expression in gastric cancer cells, consistent with the results of our previous study (10).
Expression of TP in infiltrating macrophages in relation to microvessel density in intestinal and diffuse type gastric cancer. To determine whether TP could be a specific marker enzyme of macrophages, we examined the expression of TP in tumor stromal macrophages as well as cancer cells in clinical specimens of gastric cancer using IHC. Fig. 2 shows representative $\mathrm{IHC}$ images for $\mathrm{TP}, \mathrm{CD}^{+} 8^{+}$and $\mathrm{CD} 163^{+}$macrophages and MVD in gastric cancers. TP was expressed mainly in cancer stromal cells. The numbers of infiltrating macrophages and MVD were found to be higher in cancerous than in non-cancerous regions. Box plots based on quantitative determination of the expression levels of various biomarkers by IHC analysis showed that the expression levels of TP, $\mathrm{CD}^{+} 8^{+}$macrophages, $\mathrm{CD} 163^{+}$macrophages and MVD were all similar between intestinal-type and diffuse-type gastric cancers (data not shown).

Close association of TP with infiltrating macrophages and TAMs in gastric cancer. CD68 is a known marker by which all monocytes/macrophages can be recognized. By contrast, CD163 is monocyte/macrophage-specific membrane protein that is closely associated with the anti-inflammatory M2 macrophage phenotype, and is a useful marker for distinguishing M2 macrophages that are linked to TAMs from proinflammatory M1 macrophages (18). Fig. 3 shows cross-plots between $\mathrm{TP}$ and infiltrating $\mathrm{CD}^{+} 8^{+}$and $\mathrm{CD} 163^{+}$macrophages with Spearman's rank correlation coefficents in patients with both intestinal and diffuse type gastric cancer. There was a positive correlation between TP and CD68 in both intestinal type (Spearman's rank correlation $\mathrm{r}=0.497, \mathrm{p}<0.001$ ) and diffuse type $(r=0.365, p=0.007)$ cancer. Furthermore, there 


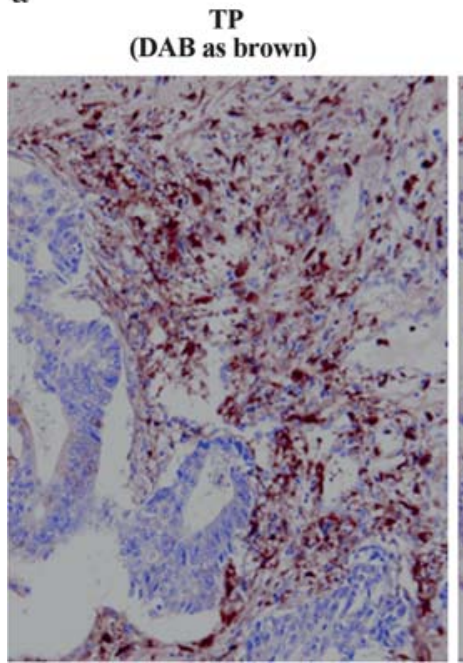

CD68 (VIP as purple)

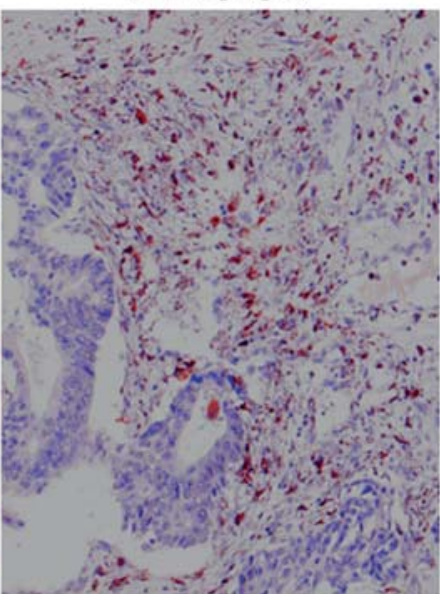

Co-expression of TP and CD68 (Pseudocolored as yellow)

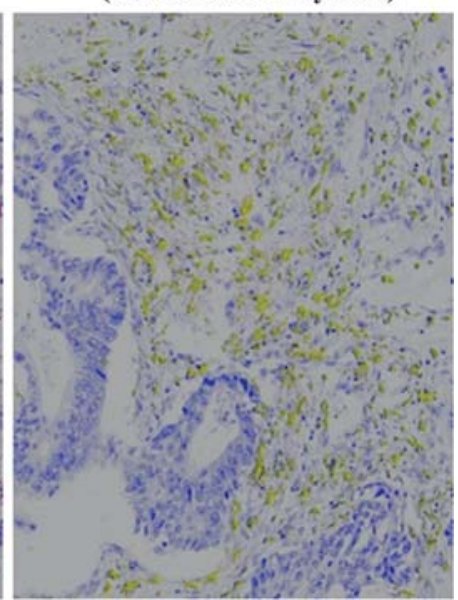

b
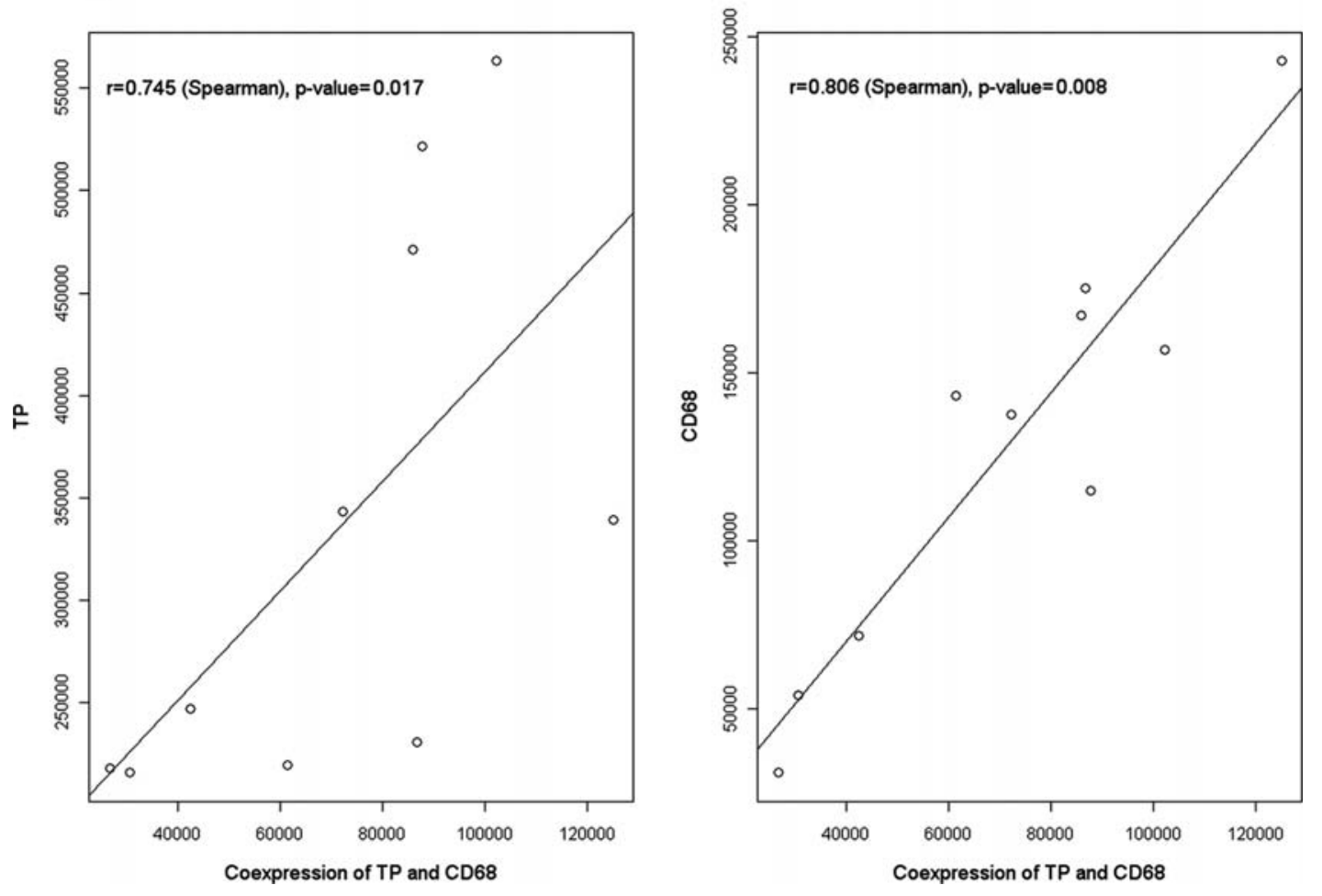

Figure 4. The quantitative analysis of close association of TP expression and CD68 $8^{+}$macrophage in IHC images of gastric cancer. CD68 macrophage was labeled with VIP (purple) and TP was labeled with DAB (brown), and the co-expression of CD68 and TP was pseudocolored as yellow (a). Panel b is a cross plot of the average co-expression area of CD68 and TP (y-axis) over the average expression area of CD68 (x-axis) was presented for 10 randomly selected patients. The average expression area was calculated from three points for each specimen (b).

was a positive correlation between TP and CD163 in both intestinal-type $(r=0.486, p<0.001)$ and diffuse type $(r=0.696$, $\mathrm{p}<0.001)$ cancer. Thus in gastric cancers there was a positive correlation between TP and infiltrating macrophages/TAMs.

Fig. 4a shows the expression of TP in macrophages in representative IHC images. TP was found to be expressed in macrophages that infiltrated gastric cancers. TP was labeled brown with $\mathrm{DAB}$, and $\mathrm{CD} 68^{+}$macrophages were labeled purple with VIP. Co-expression of TP and CD68 was pseudocolored in yellow, indicating that most macrophages expressed TP (TP-positive macrophages). Quantitative analysis of speci- mens from 10 patients indicated that the area of co-expression of CD68 and TP showed a statistically significant positive correlation with the area of expression of DAB-labeled TP $(r=0.745, p=0.017)$ and VIP-labeled CD68 $(r=0.806, p=0.008)$ (Fig. 4b).

TP and infiltrating macrophages affect tumor angiogenesis in gastric cancers. We then investigated the relationship between TP, infiltrating macrophages and MVD in gastric cancers. Fig. 5 shows cross-plots among TP, infiltrating macrophages and MVD with Spearman's rank correlation co- 
Table II. Correlation of TP, MVD and infiltrating macrophage number with lymph node metastasis, liver metastasis and peritoneal dissemination in intestinal type.

\begin{tabular}{lccc}
\hline & \multicolumn{3}{c}{ Intestinal type of gastric cancer } \\
\cline { 2 - 4 } & P-value & OR & $95 \%$ CI \\
\hline Lymph node metastasis & & & \\
TP & 0.040 & 1.898 & $(1.029,3.502)$ \\
CD68 ${ }^{+}$macrophage & 0.093 & 2.091 & $(0.885,4.943)$ \\
CD163+ macrophage & 0.003 & 4.307 & $(1.657,11.197)$ \\
MVD & 0.431 & 1.531 & $(0.530,4.417)$ \\
Liver metastasis & & & \\
TP & 0.326 & 1.426 & $(0.702,2.894)$ \\
CD68 ${ }^{+}$macrophage & 0.103 & 2.166 & $(0.856,5.481)$ \\
CD163+ macrophage & 0.856 & 0.932 & $(0.436,1.991)$ \\
MVD & 0.059 & 3.029 & $(0.959,9.564)$ \\
Peritoneal dissemination & & & \\
TP & 0.612 & 0.818 & $(0.377,1.777)$ \\
CD68 ${ }^{+}$macrophage & 0.149 & 2.299 & $(0.743,7.115)$ \\
CD163+ macrophage & 0.749 & 1.166 & $(0.456,2.979)$ \\
MVD & 0.660 & 0.714 & $(0.158,3.216)$ \\
\hline
\end{tabular}

Odds ratio (OR) and its 95\% CI are those of patients with $75 \%$ quantile of TP, macrophage number and MVD to those with $25 \%$ quantile. OR is calculated by fitting logistic regression model with TP, macrophage number and MVD, age and gender, where TP, macrophage and MVD were log-transformed.

efficients in intestinal and diffuse type gastric cancers. There was a significant positive correlation between TP and MVD in intestinal type $(\mathrm{r}=0.338, \mathrm{p}=0.010)$ gastric cancer, but not diffuse type ( $\mathrm{r}=0.209, \mathrm{p}=0.129)$ gastric cancer (Fig. 5a). There was a positive correlation between $\mathrm{CD} 68^{+}$macrophages and
MVD in both intestinal $(\mathrm{r}=0.750, \mathrm{p}<0.001)$ and diffuse type $(\mathrm{r}=0.553, \mathrm{p}<0.001)$ gastric cancer (Fig. 5b). Similarly, there was a positive correlation between $\mathrm{CD} 163^{+}$macrophages and MVD in both intestinal $(\mathrm{r}=0.307, \mathrm{p}=0.021)$ and diffuse type $(\mathrm{r}=0.272, \mathrm{p}=0.046)$ cancer (Fig. 5c). Thus, infiltrating CD68 ${ }^{+}$ and $\mathrm{CD}_{163^{+}}$macrophages are positively associated with MVD in both intestinal and diffuse type gastric cancer.

TP and infiltrating macrophages are associated with metastasis and/or survival of cancer patients. We examined whether lymph node metastasis, liver metastasis and peritoneal dissemination were associated with TP, infiltrating macrophages and MVD (Table II). In intestinal type cancer, $\mathrm{TP}(\mathrm{p}=0.040)$ and $\mathrm{CD} 163^{+}$macrophages $(\mathrm{p}=0.003)$ were significantly associated with the occurrence of lymph node metastasis. By contrast, TP, infiltrating $\mathrm{CD}^{+} 8^{+}$and $\mathrm{CD} 163^{+}$ macrophages and MVD were unassociated with metastases in diffuse type cancer (data not shown).

We also examined whether the expression of TP, infiltrating macrophages and MVD was associated with the overall survival of gastric cancer patients. P-values of the log-rank tests for TP, infiltrating macrophages and MVD are summarized in Table III. In intestinal type cancer, the association of $\mathrm{CD}^{2} 8^{+}$macrophages was statistically significant $(\mathrm{p}<0.001)$, and that of MVD was nearly significantly $(\mathrm{p}=0.060)$. Table III also gives the $\mathrm{p}$-values and hazard ratio estimates with Cox regression adjustment for age and gender as possible confounding factors. The number of $\mathrm{CD} 68^{+}$macrophages had a significant effect on overall survival $(\mathrm{p}=0.010)$, and the $\mathrm{p}$-value for MVD was close to statistical significance $(\mathrm{p}=0.060)$ even after adjusting for possible confounding factors.

Kaplan-Meier estimates of overall survival for patients with intestinal type and diffuse type cancer are presented in Fig. 6 in relation to $\mathrm{CD}^{+} 8^{+}$macrophage status. Patients with the intestinal type and an increased number of $\mathrm{CD}^{+} 8^{+}$ macrophages showed poor overall survival (Fig. 6a), whereas for patients with the diffuse type there was no apparent association of $\mathrm{CD}^{+} 8^{+}$macrophages with overall survival (Fig. 6b).

Table III. Effects of TP, infiltrating macrophage number and MVD on overall survival by log-rank test and Cox regression in gastric cancers.

\begin{tabular}{|c|c|c|c|c|c|c|c|c|}
\hline & \multicolumn{4}{|c|}{ Intestinal type } & \multicolumn{4}{|c|}{ Diffuse type } \\
\hline & \multirow{2}{*}{$\begin{array}{c}\text { Log-rank } \\
\text { test } \\
\text { P-value }\end{array}$} & \multicolumn{3}{|c|}{ Cox regression } & \multirow{2}{*}{$\begin{array}{c}\begin{array}{c}\text { Log-rank } \\
\text { test }\end{array} \\
\text { P-value }\end{array}$} & \multicolumn{3}{|c|}{ Cox regression } \\
\hline & & P-value & HR & $95 \% \mathrm{CI}$ & & P-value & HR & $95 \% \mathrm{CI}$ \\
\hline $\mathrm{TP}$ & 0.476 & 0.845 & 0.965 & $(0.678,1.375)$ & 0.380 & 0.817 & 0.935 & $(0.531,1.647)$ \\
\hline $\mathrm{CD} 68^{+}$macrophage & $<0.001$ & 0.010 & 2.045 & $(1.183,3.535)$ & 0.487 & 0.587 & 0.847 & $(0.467,1.539)$ \\
\hline $\mathrm{CD} 63^{+}$macrophage & 10.089 & 0.242 & 1.300 & $(0.838,2.017)$ & 0.918 & 0.752 & 0.926 & $(0.574,1.493)$ \\
\hline MVD & 0.060 & 0.060 & 1.938 & $(0.973,3.860)$ & 0.089 & 0.249 & 0.727 & $(0.423,1.250)$ \\
\hline
\end{tabular}

Hazard ratio (HR) and its $95 \%$ CI are those of patients with $75 \%$ quantile of TP, macrophage number and MVD to those with $25 \%$ quantile. HR is calculated by fitting Cox regression model with TP, macrophage and MVD, age and gender where TP, macrophage and MVD were log-transformed. 
a

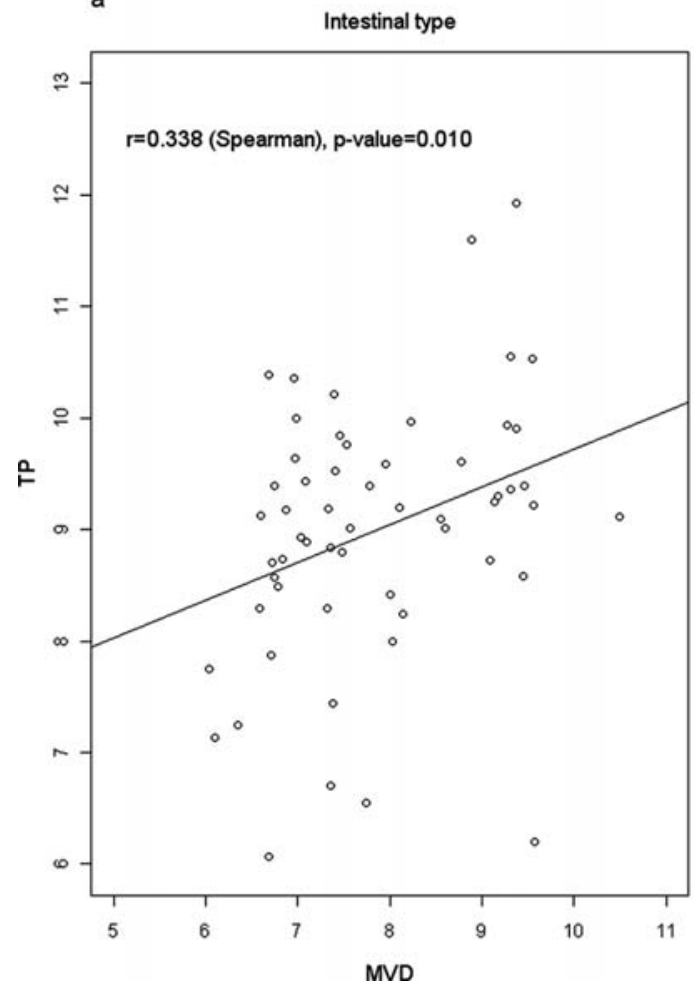

b

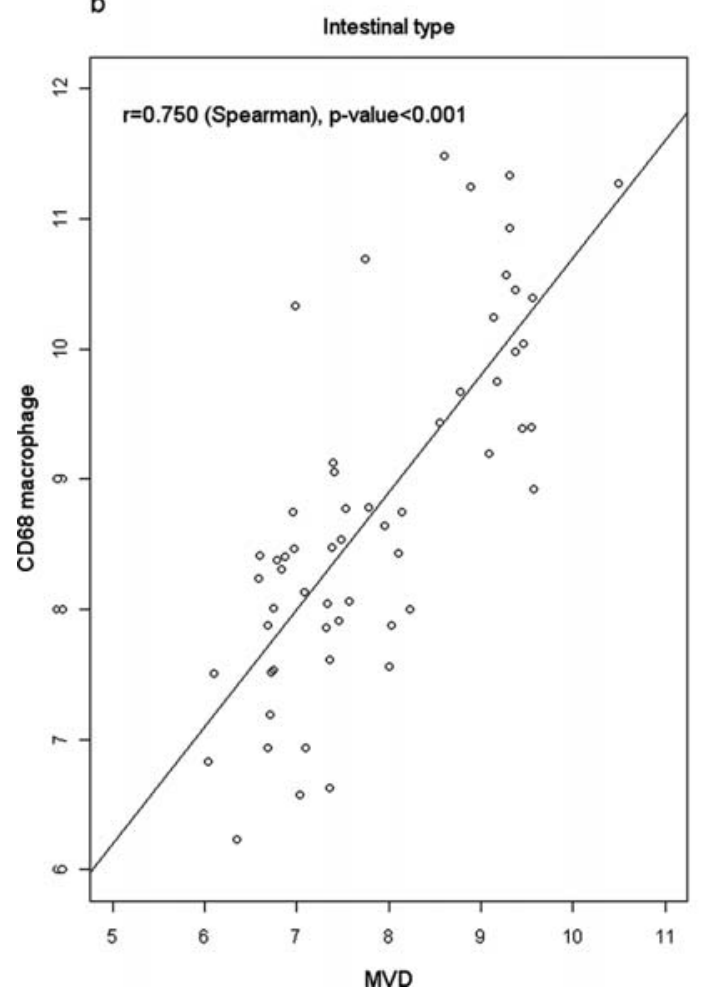

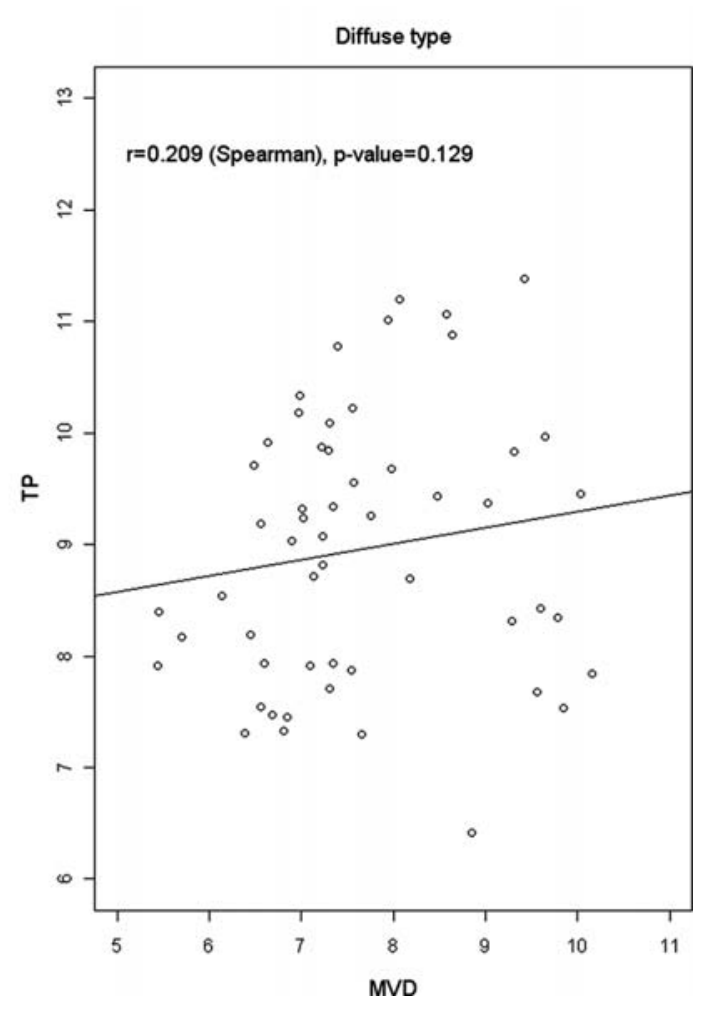

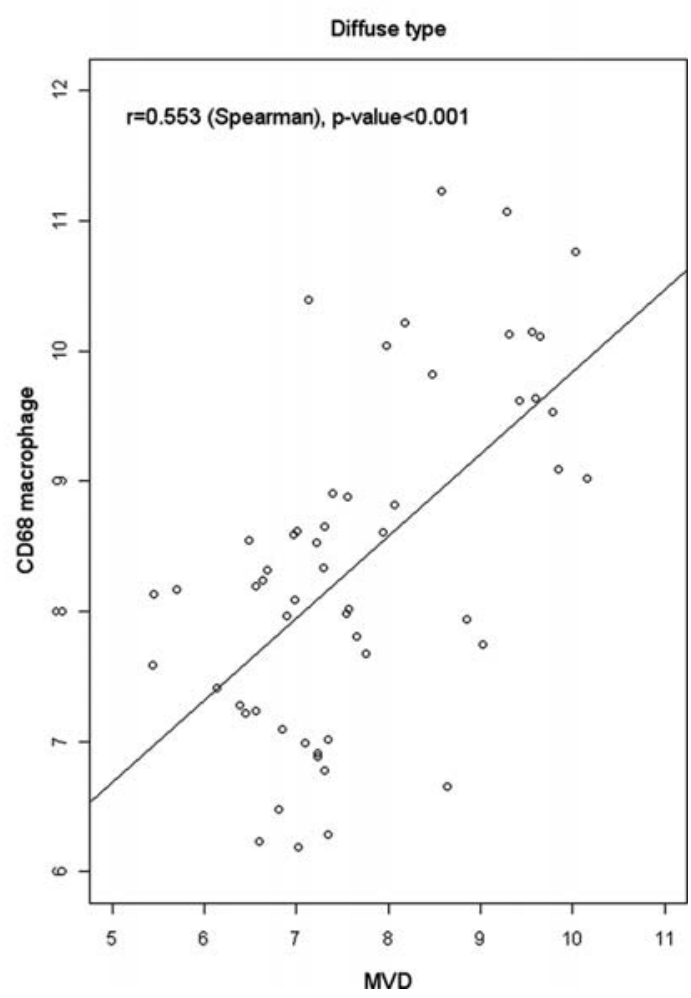

Figure 5. a and b. Correlation with MVD and TP (a) or CD68+ macrophage (b).

\section{Discussion}

In our present study, we demonstrated that expression of TP in macrophages may affect tumor angiogenesis and prognosis in patients with intestinal type gastric cancer. i) Expression of TP was observed in macrophages infiltrating both intestinal and diffuse type gastric cancer; ii) Expression of TP and the number of infiltrating macrophages were significantly associated with tumor angiogenesis in intestinal-type gastric cancer; iii) The number of infiltrating macrophages was significantly associated with overall survival in patients with intestinal-type gastric cancer.

Infiltrating macrophages were closely correlated with TP expression and tumor angiogenesis. Our present findings 

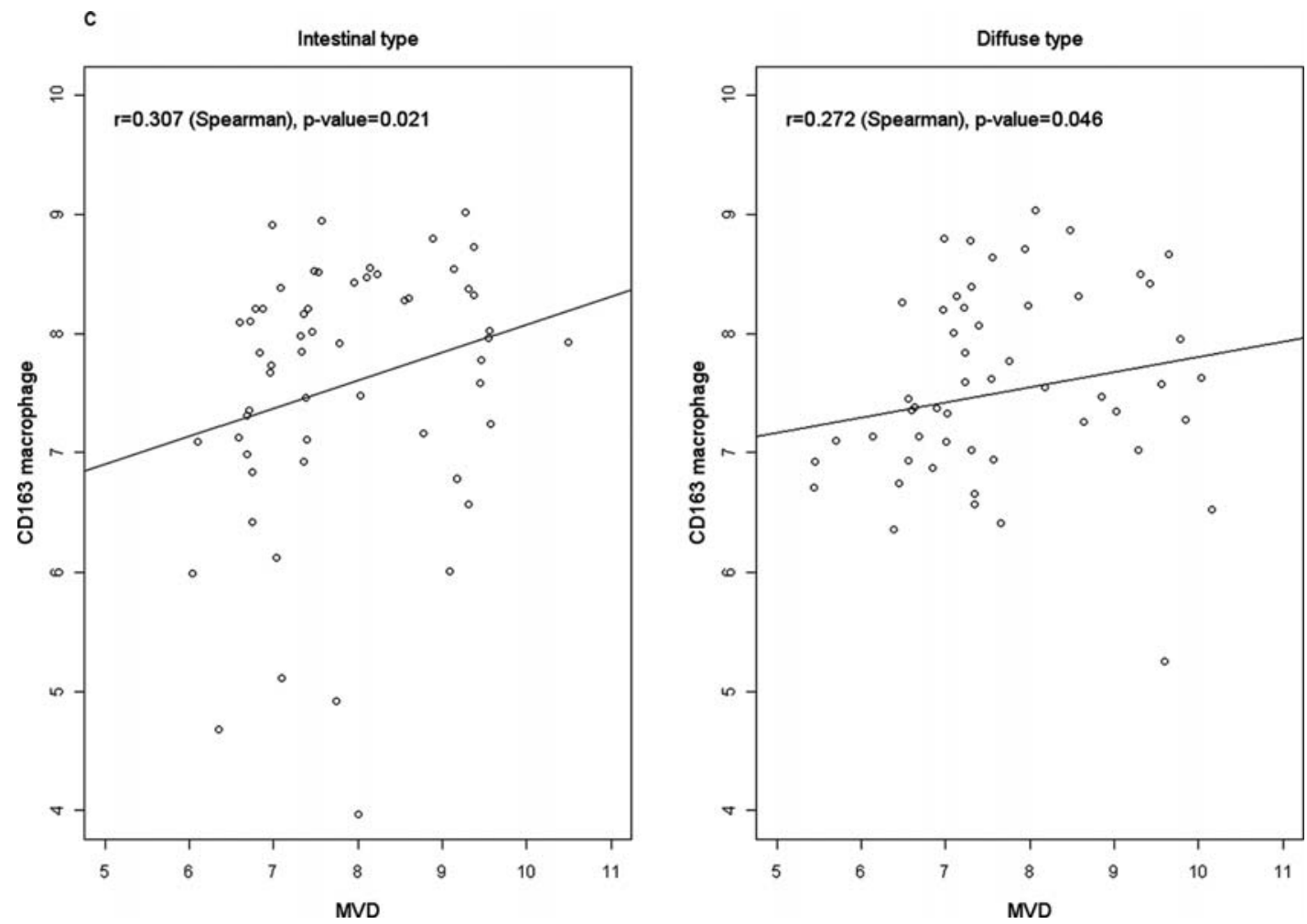

Figure 5. Correlation with MVD and TP (a) or CD68 $8^{+}$macrophage (b) or $\mathrm{CD}_{163^{+}}$macrophage (c) in intestinal type (left) and diffuse type (right) of gastric cancer.
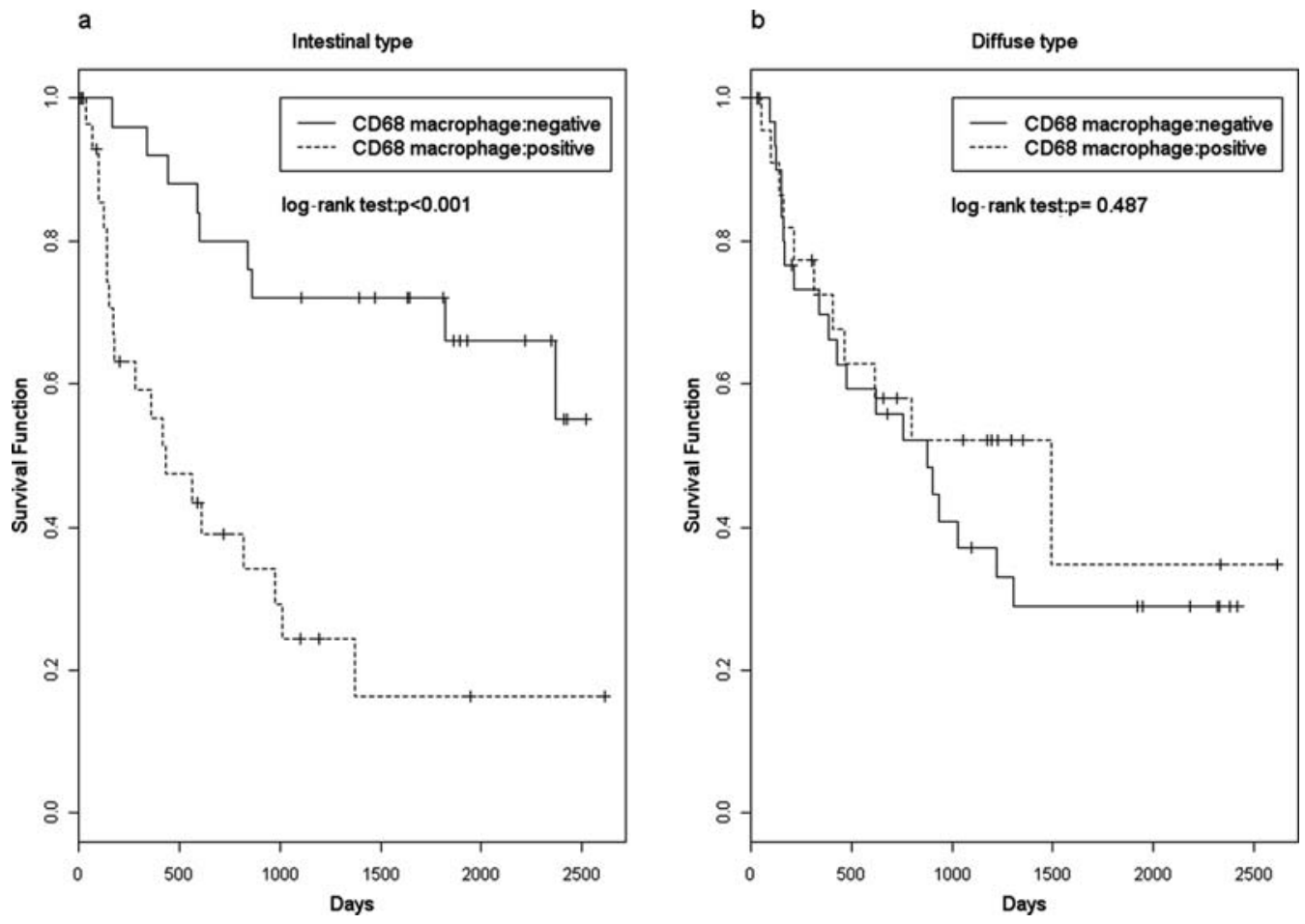

Figure 6. Kaplan-Meier estimators for overall survival in intestinal type (a) and diffuse type (b) of gastric cancer according to higher (---) and lower (-) infiltration of macrophage status with p-value by the log-rank test. In patients with higher number of infiltrating macrophages, increased number of macrophages have statistically significantly poor prognosis for overall survival.

suggest that the expression of TP in infiltrating macrophages and TAMs affects tumor angiogenesis in intestinal-type gastric cancer. The presence of extensive macrophage/TAM infiltration in tumors has often been shown to correlate with poor prognosis in cancers of the breast, cervix and bladder, and also in melanoma (19). However, the possible role of 
infiltrating macrophages/TAMs in the progression of gastric cancer remains unknown. Concerning possible predictive markers of macrophages or TAMs in gastric cancer, Migita et al have reported that the number of $\mathrm{CD}^{+} 8^{+}$macrophages tends to be associated with better patient survival, but not to a significant degree (29). Furthermore, Ohno et al have reported that the degree of $\mathrm{CD} 68^{+}$macrophage infiltration in cancer cell nests is a significant predictor of better prognosis in patients with gastric cancer (28). At present, it remains unclear why infiltration of $\mathrm{CD}^{+} 8^{+}$macrophages tends to be a marker of favorable prognosis only in gastric cancer, whereas it is a marker of poor prognosis in other human malignancies (see Introduction) (19). In the present study, however, the number of $\mathrm{CD}^{2} 8^{+}$macrophages was found to be significantly correlated with poor prognosis in patients with intestinal type cancer, but not in patients with diffuse type cancer, suggesting that infiltration of tumor-associated macrophages could affect malignant progression and prognosis according to the histological type of gastric cancer. Furthermore, tumor angiogenesis was also closely associated with infiltration of CD68 ${ }^{+}$ or $\mathrm{CD}_{163^{+}}$macrophages (Fig. 5). Based on these findings, we consider that infiltration of macrophages/TAMs affects the prognosis of intestinal type gastric cancer. However, a more detailed study will be necessary to clarify how tumorassociated macrophages differentially modulate prognosis or malignant progression in intestinal-type and diffuse-type gastric cancer.

Akiyama and colleagues have previously reported rather specific expression of TP in macrophages relative to cancer cells in differentiated gastric adenocarcinoma $(4,8)$, and patients with TP-positive carcinomas have a poorer prognosis than those with TP-negative differentiated adenocarcinomas. Our present study also demonstrated that tumor-infiltrating macrophages are a potent prognostic marker in intestinaltype, but not diffuse-type, gastric cancer. Inflammatory stimuli markedly enhance the expression of TP in macrophages through transcriptional activation (Fig. 1) (10). Expression of TP results in enhanced production of reactive oxygen species by 2-deoxy-D-ribose, a downstream mediator of TP, indicating that TP induces oxygen stress $(2,4,34)$. TP-positive macrophages are thus expected to be highly active, not only in the production of reactive oxygen, but also various angiogenesis factors, growth factors, chemokines and proteases, indicating that such macrophages are highly angiogenic. In fact, through cross-talk with cancer cells, macrophages are induced to undergo transformation into tumor-supporting macrophages that actively stimulate angiogenesis and cancer cell invasion $(13,14,20)$. Thus, the expression of TP in macro-phages might play a key role in angiogenesis, tumor growth and anti-apoptosis in malignant cancers in the stomach as well as those of the colon and other organs, possibly through the enhanced production of angiogenesis factors by macrophages $(2,4)$. In both intestinal and diffuse type gastric cancer, the number of macrophages was significantly correlated with tumor angiogenesis, and TP expression was also positively correlated with the presence of both $\mathrm{CD}^{+} 8^{+}$and $\mathrm{CD} 163^{+}$macrophages. By contrast, TP expression was significantly associated with angiogenesis only in intestinal type gastric cancer, and not in the diffuse type. A further study will be needed to clarify why TP expression thus differentially affects angiogenesis in gastric cancer depending on its histological type.

In conclusion, our present study demonstrates a close association of TP expression in tumor-infiltrating macrophages with angiogenesis in gastric cancer, lending support to the idea that TP-positive macrophages promote angiogenesis and metastasis in this cancer. TP expression in macrophages could be a useful and novel biomarker for diagnosis of gastric cancer and the development of new therapeutic strategies.

\section{Acknowledgements}

This research was supported by a grant-in-aid for Scientific Research for Priority areas, Cancer, from the Ministry of Education Culture, Sports, Science and Technology of Japan (M.O.), and by the 3rd Term Comprehensive Control Research for Cancer from the Ministry of Health, Labor and Welfare, Japan (M. Ku.). This study was also supported in part by the Formation of the Innovation Center for Fusion of Advanced Technologies, Kyushu University, Japan (M.O., M. Ku.). We thank Takashi Yanagawa (Kurume University) for fruitful discussion on biostatistical analysis.

\section{References}

1. Miyadera K, Sumizawa T, Haraguchi M, et al: Role of thymidine phosphorylase activity in the angiogenic effect of platelet derived endothelial cell growth factor/thymidine phosphorylase. Cancer Res 55: 1687-1690, 1995.

2. Brown NS, Jones A, Fujiyama C, Harris AL and Bicknell R: Thymidine phosphorylase induces carcinoma cell oxidative stress and promotes secretion of angiogenic factors. Cancer Res 60: 6298-6302, 2000.

3. Uchimiya H, Furukawa T, Okamoto M, et al: Suppression of thymidine phosphorylase-mediated angiogenesis and tumor growth by 2-deoxy-L-ribose. Cancer Res 62: 2834-2839, 2002.

4. Akiyama S, Furukawa T, Sumizawa T, et al: The role of thymidine phosphorylase, an angiogenic enzyme, in tumor progression. Cancer Sci 95: 851-857, 2004.

5. Yoshimura A, Kuwazuru Y, Furukawa T, Yoshida H, Yamada K and Akiyama S: Purification and tissue distribution of human thymidine phosphorylase; high expression in lymphocytes, reticulocytes and tumors. Biochim Biophys Acta 1034: 107-113, 1990.

6. O'Brien T, Cranston D, Fuggle S, Bicknell R and Harris AL: Different angiogenic pathways characterize superficial and invasive bladder cancer. Cancer Res 55: 510-513, 1995.

7. Takebayashi Y, Akiyama S, Akiba S, et al: Clinicopathologic and prognostic significance of an angiogenic factor, thymidine phosphorylase, in human colorectal carcinoma. J Natl Cancer Inst 88: 1110-1117, 1996.

8. Shimaoka S, Matsushita S, Nitanda T, et al: The role of thymidine phosphorylase expression in the invasiveness of gastric carcinoma. Cancer 88: 2220-2227, 2000.

9. Takao S, Takebayashi Y, Che X, et al: Expression of thymidine phosphorylase is associated with a poor prognosis in patients with ductal adenocarcinoma of the pancreas. Clin Cancer Res 4: 1619-1624, 1998

10. Goto H, Kohno K, Sone S, Akiyama S, Kuwano M and Ono M: Interferon gamma-dependent induction of thymidine phosphorylase/platelet-derived endothelial growth factor through gammaactivated sequence-like element in human macrophages. Cancer Res 61: 469-473, 2001.

11. Torisu H, Ono M, Kiryu H, et al: Macrophage infiltration correlates with tumor stage and angiogenesis in human malignant melanoma: possible involvement of TNF $\alpha$ and IL-1 $\alpha$. Int J Cancer 85: 182-188, 2000.

12. Torisu-Itakura H, Furue M, Kuwano M and Ono M: Coexpression of thymidine phosphorylase and heme oxygenase-1 in macrophages in human malignant vertical growth melanomas. Jpn J Cancer Res 91: 906-910, 2000. 
13. Pollard JW: Tumor-educated macrophages promote tumor progression and metastasis. Nat Rev Cancer 4: 71-78, 2004.

14. Ono M: Molecular links between tumor angiogenesis and inflammation: inflammatory stimuli of macrophages and cancer cells as targets for therapeutic strategy. Cancer Sci 99: 1501-1506, 2008 .

15. Mantovani A, Sica A, Sozzani S, Allavena P, Vecchi A and Locati M: The chemokine system and diverse forms of macrophage activation and polarization. Trends Immunol 25: 677-686, 2004.

16. Martinez FO, Gordon S, Locati M and Mantovani A: Transcriptional profiling of the human monocyte-to-macrophage differentiation and polarization: new molecules and patterns of gene expression. J Immunol 177: 7303-7311, 2006.

17. Forssell J, Oberg A, Henriksson ML, Stenling R, Jung A and Palmqvist R: High macrophage infiltration along the tumor front correlates with improved survival in colon cancer. Clin Cancer Res 13: 1472-1479, 2007.

18. Komohara Y, Ohnishi K, Kuratsu J and Takeya M: Possible involvement of the M2 anti-inflammatory macrophage phenotype in growth of human gliomas. J Pathol 216: 15-24, 2008.

19. Bingle L, Brown NJ and Lewis CE: The role of tumor-associated macrophages in tumor progression: implications for new anticancer therapies. J Pathol 196: 254-265, 2002.

20. Lewis CE and Pollard JW: Distinct role of macrophages in different tumor microenvironments. Cancer Res 66: 605-612, 2006.

21. Nakao S, Kuwano T, Tsutsumi-Miyahara C, et al: Infiltration of COX-2-expressing macrophages is a prerequisite for IL- 1 betainduced neovascularization and tumor growth. J Clin Invest 115: 2979-2991, 2005

22. Kimura YN, Watari K, Fotovati A, et al: Inflammatory stimuli from macrophages and cancer cells synergistically promote tumor growth and angiogenesis. Cancer Sci 98: 2009-2018, 2007.

23. Hiraoka K, Zenmyo M, Watari K, et al: Inhibition of bone and muscle metastases of lung cancer cells by a decrease in the number of monocytes/macrophages. Cancer Sci 99: 1595-1602, 2008
24. Yoshimura A: Signal transduction of inflammatory cytokines and tumor development. Cancer Sci 97: 439-447, 2006.

25. Sica A and Bronte V: Altered macrophage differentiation and immune dysfunction in tumor development. J Clin Invest 117: 1155-1166, 2007.

26. Lin EY and Pollard JW: Tumor-associated macrophages press the angiogenic switch in breast cancer. Cancer Res 67: 5064-5066, 2007.

27. Nishie A, Ono M, Shono T, et al: Macrophage infiltration and heme oxygenase-1 expression correlate with angiogenesis in human gliomas. Clin Cancer Res 5: 1107-1113, 1999.

28. Ohno S, Inagawa H, Dhar DK, et al: The degree of macrophage infiltration into the cancer cell nest is a significant predictor of survival in gastric cancer patients. Anticancer Res 23: 5015-5022, 2003.

29. Migita T, Sato E, Saito K, et al: Differing expression of MMPs-1 and -9 and urokinase receptor between diffuse and intestinaltype gastric carcinoma. Int J Cancer 84: 74-79, 1999.

30. Lauren P: The two histological main types of gastric carcinoma: diffuse and so-called intestinal-type carcinoma. An attempt at a histo-clinical classification. Acta Pathol Microbiol Scand 64: 31-49, 1965.

31. Sugano H, Nakamura $\mathrm{K}$ and Kato $\mathrm{Y}$ : Pathological studies of human gastric cancer. Acta Pathol Jpn 32: 329-347, 1982.

32. Xu X, Gimotty PA, Guerry D, et al: Lymphatic invasion revealed by multispectral imaging is common in primary melanomas and associates with prognosis. Hum Pathol 39: 901-909, 2008.

33. Fujii T, Kawahara A, Basaki Y, et al: Expression of HER2 and estrogen receptor alpha depends upon nuclear localization of Y-box binding protein-1 in human breast cancers. Cancer Res 68: 1504-1512, 2008

34. Brown NS and Bicknell R: Thymidine phosphorylase, 2-deoxyD-ribose and angiogenesis. Biochem J 334: 1-8, 1998. 